\title{
Finite surgeries on three-tangle pretzel knots
}

\author{
DAVID FUTER \\ MASAHARU ISHIKAWA \\ YUICHI KABAYA \\ THOMAS W MATTMAN \\ KOYA SHIMOKAWA
}

\begin{abstract}
We classify Dehn surgeries on $(p, q, r)$ pretzel knots that result in a manifold of finite fundamental group. The only hyperbolic pretzel knots that admit nontrivial finite surgeries are $(-2,3,7)$ and $(-2,3,9)$. Agol and Lackenby's 6 -theorem reduces the argument to knots with small indices $p, q, r$. We treat these using the Culler-Shalen norm of the $\operatorname{SL}(2, \mathbb{C})$-character variety. In particular, we introduce new techniques for demonstrating that boundary slopes are detected by the character variety.
\end{abstract}

57M05, 57M25, 57M50

Dedicated to Professor Akio Kawauchi on the occasion of his 60th birthday.

In [19] Mattman showed that if a hyperbolic $(p, q, r)$ pretzel knot $K$ admits a nontrivial finite Dehn surgery of slope $s$ (ie, a Dehn surgery that results in a manifold of finite fundamental group) then either

- $K=(-2,3,7)$ and $s=17,18$ or 19 ,

- $K=(-2,3,9)$ and $s=22$ or 23 or

- $K=(-2, p, q)$ where $p$ and $q$ are odd and $5 \leq p \leq q$.

In the current paper we complete the classification by proving:

Theorem 1 Let $K$ be a $(-2, p, q)$ pretzel knot with $p, q$ odd and $5 \leq p \leq q$. Then $K$ admits no nontrivial finite surgery.

Using the work of Agol [1] and Lackenby [16], candidates for finite surgery correspond to curves of length at most six in the maximal cusp of $S^{3} \backslash K$. If $7 \leq p \leq q$, we will argue that only five slopes for the $(-2, p, q)$ pretzel knot have length six or less: the meridian and the four integral surgeries $2(p+q)-1,2(p+q), 2(p+q)+1$ and $2(p+q)+2$. If $p=5$ and $q \geq 11$, a similar argument leaves seven candidates, the meridian and the six integral slopes between $2(5+q)-2$ and $2(5+q)+3$. 
We will treat the remaining knots, $(-2,5,5),(-2,5,7)$ and $(-2,5,9)$, using the Culler-Shalen norm (for example, see Boyer and Zhang [2] and Culler, Gordon, Luecke and Shalen [5]). For a hyperbolic knot in $S^{3}$, this is a norm $\|\cdot\|$ on the vector space $H_{1}(\partial M ; \mathbb{R})$. We can identify a Dehn surgery slope $s \in \mathbb{Q} \cup\left\{\frac{1}{0}\right\}$ with a class $\gamma_{s} \in H_{1}(\partial M ; \mathbb{Z})$. If $s$ is a finite slope that is not a boundary slope, the finite surgery theorem [2] shows that $s$ is integral or half-integral and $\left\|\gamma_{s}\right\| \leq \max \{2 S, S+8\}$ where $S=\min \left\{\|\gamma\|: 0 \neq \gamma \in H_{1}(\partial M ; \mathbb{Z})\right\}$ is the minimal norm. This makes the Culler-Shalen norm an effective tool for the study of finite surgery slopes.

The Culler-Shalen norm is intimately related to the set of boundary slopes. An essential surface in the knot complement $M$ will meet $\partial M$ in a (possibly empty) set of parallel curves. The slope represented by this set of curves is known as a boundary slope. For a pretzel knot, these slopes are determined by the algorithm of Hatcher and Oertel [11]. Given the list of boundary classes $\left\{\beta_{j}: 1 \leq j \leq N\right\}$, the norm is determined by an associated set of nonnegative integers $a_{j}$ :

$$
\|\gamma\|=2 \sum_{j=1}^{N} a_{j} \Delta\left(\gamma, \beta_{j}\right) .
$$

Here $\Delta(\cdot, \cdot)$ denotes the minimal geometric intersection number.

In particular, if a boundary class $\beta_{j}$ is detected by the character variety, then the corresponding coefficient $a_{j}$ is positive. To describe the notion of "detection," recall that, following Culler and Shalen [6], we can use $\operatorname{SL}(2, \mathbb{C})$-representations of the knot group to construct essential surfaces in the complement $M$. The construction uses an ideal point of a curve in the $\operatorname{SL}(2, \mathbb{C})$-character variety to induce a nontrivial action of the knot group on a Bass-Serre tree. This action in turn yields an embedded essential surface in $M$. We will say that the boundary slope associated to a surface so constructed is detected by the character variety. As it will be enough for us to argue that certain $a_{j}$ are positive, an important part of our proof is the introduction of new techniques to show that a boundary slope is detected.

We remark that Ichihara and Jong have recently announced an independent proof of Theorem 1 using Heegaard Floer homology [12]. Indeed, they go further and classify finite and cyclic surgeries for all Montesinos knots. Also, with an argument based on Khovanov homology, Watson [27] has just shown that the $(-2, p, p)$ pretzel knot (for $p$ odd, $5 \leq p \leq 25$ ) admits no nontrivial finite surgeries.

Our paper is organised as follows. As above, our proof of Theorem 1 breaks into two cases. In Section 4, we use the 6-theorem to handle the case where $p \geq 7$ as well as the case where $p=5$ and $q \geq 11$ and prove that none of these knots admit nontrivial finite 
surgeries. This leaves the three knots $(-2,5,5),(-2,5,7)$ and $(-2,5,9)$, which we treat using the Culler-Shalen norm. In Section 2, we show that the $(-2,5,5)$ pretzel knot admits no nontrivial finite surgeries by introducing new techniques for detecting boundary slopes that generalise the method introduced by Kabaya [14] (based on earlier work of Yoshida). In Section 3, we give some observations concerning detection of the boundary slopes $2(p+q)$ for the $(-2,5,5)$ and $(-2,5,7)$ pretzel knots using techniques pioneered by Ohtsuki $[22 ; 23]$. In consequence, we conclude that the $(-2,5,7)$ pretzel knot admits no nontrivial finite surgeries. We conclude Section 3 by proving the same assertion for the $(-2,5,9)$ pretzel knot. Thus, in each section, we show that the knots under consideration admit no nontrivial finite slopes. Taken together, this proves Theorem 1.

In the next section we collect some general results that will be used throughout the paper.

\section{Lemmas}

In this section let $K$ denote a $(-2, p, q)$ pretzel knot with $p, q$ odd and $5 \leq p \leq q$. We collect several facts about finite slopes of these knots. We begin with arguments that apply to all slopes. We next look at arguments specific to even integer slopes and those that apply to the slopes $2(p+q) \pm 1$.

For a pair of slopes $\frac{a}{b}, \frac{c}{d} \in \mathbb{Q} \cup\left\{\frac{1}{0}\right\}$, the distance is defined to be $\Delta\left(\frac{a}{b}, \frac{c}{d}\right)=|a d-b c|$. This is equivalent to the minimal geometric intersection number of curves representing these two slopes. Agol [1] and Lackenby [16] independently showed that any pair of exceptional slopes on a one-cusped hyperbolic manifold lie within distance 10 of each other. Very recently, Lackenby and Meyerhoff [17] improved the bound from 10 to 8; we will not need this improvement.

Since $2(p+q)$ is an exceptional, toroidal surgery slope of the $(-2, p, q)$ pretzel knot $K$ [29], it follows from the work of Agol [1] and Lackenby [16] that any other exceptional slope $s$ is within distance 10 of $2(p+q)$.

Lemma 2 Let $p, q$ be odd and $5 \leq p \leq q$. If $s$ is a finite slope of the $(-2, p, q)$ pretzel knot, then $\Delta(s, 2(p+q)) \leq 10$.

The next set of lemmas relate to the Culler-Shalen norm for a hyperbolic knot in $S^{3}$; Shalen [25] is a good reference. Note that $K$ is hyperbolic [15]. We will be working with the total norm, which is given by summing the Culler-Shalen norm over all components of the character variety. The following lemma, which is proved in Section 5.1 of [18] (cf [19, Proposition 1.3]), gives the minimal total norm. 
Lemma 3 Let $p, q$ be odd with $5 \leq p \leq q$. The minimal total norm of the $(-2, p, q)$ pretzel knot is $S=2 p q-3(p+q)$.

The next lemma summarises several consequences of the finite surgery theorem [2].

Lemma 4 Let $p, q$ be odd with $5 \leq p \leq q$ and let $s=\frac{a}{b}$ be a finite surgery slope of the $(-2, p, q)$ pretzel knot. Then, either $s=\frac{1}{0}$ or else $s$ is integral (ie, $b=1$ ) or half-integral $(b=2)$. Moreover, if $\gamma_{s}$ is the class of a curve representing slope $s$ and $s$ is not a boundary slope, then $\left\|\gamma_{s}\right\| \leq S+8$ unless $s$ is an even integer, in which case $\left\|\gamma_{s}\right\| \leq 2 S$.

Note that $M\left(\frac{1}{0}\right)=S^{3}$ is known as trivial surgery and $\frac{1}{0}$ is always a finite slope. Our goal in this paper is to show that it is the only finite slope of $K$.

In earlier work, Ishikawa, Mattman and Shimokawa showed that there is a relationship between the positions of finite slopes and boundary slopes [13]. Recall that a surface $F \subset M$ is called a semifibre if its complement $M \backslash F$ is a (possibly twisted) $I$-bundle. (Under this definition, a fibre in a fibration of $M$ is one example of a semifibre.) A boundary slope is strict if it is not the slope of a semifibre of $S^{3} \backslash K$. For a knot in $S^{3}$, if $r$ is a boundary slope that is not strict, then $r=0$.

Lemma 5 Let $K$ be a hyperbolic knot in $S^{3}$ and $\|\cdot\|$ the associated total CullerShalen norm. Let $S$ denote the minimal total norm and suppose that $S \geq 8$. If $s=\frac{a}{b}$ is a finite slope of $K$, then there is a strict boundary slope $r$ with $|s-r| \leq 2 / b$ and the inequality is strict unless $K$ has exactly two strict boundary slopes detected by the character variety.

Proof In the proof of Corollary 3(2) of [13], it was shown that $|s-r| \leq 3 / b$ using $S \geq 4$. If we instead assume $S \geq 8$, the same argument shows that $|s-r| \leq 2 / b$, as required.

Finally, we observe the following immediate consequence of [5, Theorem 2.0.3]. Recall that a knot is small if there is no closed essential surface in its complement. In particular, the $(-2, p, q)$ pretzel knot $K$ is small [21].

Lemma 6 Let $\beta$ be a boundary slope for a small knot $K$ in $S^{3}$. Then $\beta$ is not a finite slope. 


\subsection{Even surgeries}

In this subsection we will see that even integer surgeries of the $(-2, p, q)$ pretzel knot are generally not finite.

Using the Wirtinger presentation [24], the fundamental group of the $(-2, p, q)$ pretzel knot is (cf [26])

$$
\begin{gathered}
\pi_{1}(M)=\langle x, y, z|(z x)^{(p-1) / 2} z(z x)^{(1-p) / 2}=(y x)^{-(q+1) / 2} y(y x)^{(q+1) / 2}, \\
\left(y z^{-1}\right)^{-1} y\left(y z^{-1}\right)=(y x)^{(1-q) / 2} x(y x)^{(q-1) / 2}, \\
\left.\left(y z^{-1}\right)^{-1} z\left(y z^{-1}\right)=(z x)^{(p+1) / 2} x(z x)^{-(p+1) / 2}\right\rangle .
\end{gathered}
$$

There is redundancy in the relations as any one is a consequence of the other two.

For an integral surgery slope $s$, the group of $M(s)$ is given by adding the relator $x^{s} l$ where

$$
l=x^{-2(p+q)}(y x)^{(q-1) / 2}\left(y z^{-1}\right)^{-1}(y x)^{(q+1) / 2}(z x)^{(p-1) / 2}\left(y z^{-1}\right)(z x)^{(p+1) / 2},
$$

represents the longitude, ie, $\pi_{1}(M(s))=\pi_{1}(M) /\left\langle x^{s} l\right\rangle$.

Lemma 7 Let $p, q$ be odd with $5 \leq p \leq q$. Assume further that if $p=5$, then $q \geq 11$. Let $s$ be an even integer and let $M$ be the complement of the $(-2, p, q)$ pretzel knot. Then $\pi_{1}(M(s))$ is not finite.

Proof We will argue that $\pi_{1}(M(s))$ projects onto the group that Coxeter [4] calls $(2, p, q ; 2)$. Edjvet [8] has shown that this group is infinite under our hypotheses on $p$ and $q$.

Assume that $s$ is even. Adding the relators $x^{2}, y^{2}, z^{2}$ and $(y z)^{2}$, we see that $\pi_{1}(M(s))$ has as factor group

$$
\begin{aligned}
& \langle x, y, z| x^{2}, y^{2}, z^{2},(y z)^{2},(z x)^{p},(y x)^{q}, \\
& \left.\quad(y x)^{(q-1) / 2}(z y)(y x)^{(q+1) / 2}(z x)^{(p-1) / 2}(y z)(z x)^{(p+1) / 2}\right\rangle .
\end{aligned}
$$

Let $G_{\mathrm{ev}}$ denote the subgroup consisting of words of even length. By substituting $a=z x, b=x y, c=y z$ we have the closely related group

$$
G_{\mathrm{ev}}^{\prime}=\left\langle a, b, c \mid c^{2}, a^{p}, b^{q}, a b c, b^{(q+1) / 2} c b^{(q-1) / 2} a^{(p-1) / 2} c a^{(p+1) / 2}\right\rangle .
$$

That is, $G_{\mathrm{ev}}$ is a quotient $F_{a, b, c} / K_{0}$ of the free group on $a, b, c$ where $K_{0}$ is the normal closure of the given relations in the free group on $x, y, z$. On the other hand, $G_{\mathrm{ev}}^{\prime}=F_{a, b, c} / K_{1}$ where $K_{1}$ is the normal closure of the relations in $F_{a, b, c}$. Evidently $K_{1} \leq K_{0}$ and it will suffice to argue that $G_{\mathrm{ev}}^{\prime}$ is infinite. 
Letting $\alpha=a^{(p-1) / 2}, \beta=b^{(q-1) / 2}$ we have

$$
\left\langle\alpha, \beta \mid \alpha^{p}, \beta^{q},\left(\alpha^{2} \beta^{2}\right)^{2},(\beta \alpha)^{2}(\alpha \beta)^{2}\right\rangle .
$$

Finally adding the relator $(\alpha \beta)^{2}$, we arrive at

$$
(2, p, q ; 2)=\left\langle\alpha, \beta \mid \alpha^{p}, \beta^{q},(\alpha \beta)^{2},\left(\alpha^{2} \beta^{2}\right)^{2}\right\rangle .
$$

\section{$1.22(p+q) \pm 1$ surgeries}

We will show that $2(p+q) \pm 1$ surgery results in a manifold with infinite fundamental group provided $p$ and $q$ are sufficiently large.

Lemma 8 Let $p, q$ be odd with $5 \leq p \leq q$ and $(p, q) \neq(5,5)$. Let $M$ be the complement of the $(-2, p, q)$ pretzel knot. Then $\pi_{1}(M(2(p+q)-1))$ is not finite.

Proof As in the previous subsection, $\pi_{1}(M(2(p+q)-1))$ is

$$
\begin{aligned}
\langle x, y, z|\left(y z^{-1}\right)^{-1} y\left(y z^{-1}\right) & =(y x)^{(1-q) / 2} x(y x)^{(q-1) / 2}, \\
\left(y z^{-1}\right)^{-1} z\left(y z^{-1}\right) & \left.=(z x)^{(p+1) / 2} x(z x)^{-(p+1) / 2}, x^{2(p+q)-1} l\right\rangle .
\end{aligned}
$$

We will write the group in terms of the generators $a=z x$ and $b=y x$. Then $y z^{-1}=$ $b a^{-1}$. It will be convenient to use the expressions $\alpha=a^{(p-1) / 2}$ and $\beta=b^{(q-1) / 2}$.

The relator $x^{2(p+q)-1} l$ allows us to write $x$ in terms of $a$ and $b: x=\beta a \beta \alpha b \alpha$. Then, $y=b x^{-1}=b(\beta a \beta \alpha b \alpha)^{-1}$ while $z=a x^{-1}=a(\beta a \beta \alpha b \alpha)^{-1}$. Using these substitutions, the first relation becomes the relator $a \beta b^{-1} a \beta(\alpha b \alpha \beta)^{2}$ while the second yields $\alpha b a^{-1} \alpha b(\alpha \beta a \beta)^{2}$. Thus, $\pi_{1}(M(2(p+q)-1))$ can be written

$$
\left\langle a, b, \alpha, \beta \mid \alpha=a^{(p-1) / 2}, \beta=b^{(q-1) / 2}, a \beta b^{-1} a \beta(\alpha b \alpha \beta)^{2}, \alpha b a^{-1} \alpha b(\alpha \beta a \beta)^{2}\right\rangle .
$$

As we shall see, by adding the relators $a^{p}, b^{q}$ and $\left(\alpha \beta^{-1}\right)^{2}$ we obtain $G^{5, p, q}$ (see Coxeter [4]) as a factor group. Since this group is infinite [9], we deduce that $\pi_{1}(M(2(p+q)-1))$ is, likewise, infinite.

Note that $a^{p}=a \alpha^{2}$ so that $a=\alpha^{-2}$. Similarly $b=\beta^{-2}$. The relator $\left(\alpha \beta^{-1}\right)^{2}$ gives $\alpha \beta^{-1}=\beta \alpha^{-1}$ and $\alpha^{-1} \beta=\beta^{-1} \alpha$. Then, the relators of $\pi_{1}(M(2(p+q)-1))$ become $\left(\beta^{-1}\left(\alpha^{2} \beta^{-2}\right)^{2}\right)^{2}$ and $\left(\left(\alpha^{2} \beta^{-2}\right)^{2} \alpha\right)^{2}$. Thus, we can write the factor group as

$$
G=\left\langle\alpha, \beta \mid \alpha^{p}, \beta^{q},\left(\alpha \beta^{-1}\right)^{2},\left(\beta^{-1}\left(\alpha^{2} \beta^{-2}\right)^{2}\right)^{2},\left(\left(\alpha^{2} \beta^{-2}\right)^{2} \alpha\right)^{2}\right\rangle .
$$

Replacing $\alpha$ by $A, \beta$ by $B^{-1}$, and introducing the generator $C=\left(A^{2} B^{2}\right)^{2}$, we can rewrite $G$ as

$$
G=\left\langle A, B, C \mid A^{p}, B^{q},(A B)^{2}(B C)^{2},(C A)^{2}, C=\left(A^{2} B^{2}\right)^{2}\right\rangle .
$$


We next show that $C^{5}$ is also a relator in this group. Since $(B C)^{2}$ is a relator, so too is $\left(C^{-1} B^{-1}\right)^{2}$. But

$$
C^{-1} B^{-1}=\left(B^{-2} A^{-2}\right)^{2} B^{-1}=B^{-1}\left(B^{-1} A^{-2} B^{-1}\right)^{2}=B^{-1}\left(A B^{2} A\right)^{2} .
$$

It follows that $\left(A B^{2} A\right)^{-2}=B^{-1}\left(A B^{2} A\right)^{2} B^{-1}$.

On the other hand, since

$$
1=(C A)^{2}=\left(\left(A^{2} B^{2}\right)^{2} A\right)^{2}=\left(A\left(A B^{2} A\right)^{2}\right)^{2}
$$

we have $\left(A B^{2} A\right)^{-2}=A\left(A B^{2} A\right)^{2} A$. Equating these two expressions for $\left(A B^{2} A\right)^{-2}$ gives the desired result, $1=\left(A^{2} B^{2}\right)^{10}=C^{5}$.

Note that $(A B C)^{2}$ is also a consequence of the relators we already know. Indeed,

$$
\begin{aligned}
(A B C)^{2} & =A B C A B C \\
& =B^{-1} A^{-1} A^{-1} C^{-1} C^{-1} B^{-1} \\
& =B^{-1} A^{-2} C^{-2} B^{-1} \\
& =B^{-1} A^{-2}\left(A^{2} B^{2}\right)^{-4} B^{-1} \\
& =B\left(A^{2} B^{2}\right)^{-5} B^{-1}=1 .
\end{aligned}
$$

Thus, we will not change the group by adding the relators $C^{5}$ and $(A B C)^{2}$ : $G=\left\langle A, B, C \mid A^{p}, B^{q}, C^{5},(A B)^{2},(B C)^{2},(C A)^{2},(A B C)^{2}, C=\left(A^{2} B^{2}\right)^{2}\right\rangle$.

Finally, we note that $C=\left(A^{2} B^{2}\right)^{2}$ is now a consequence of the other relators. Indeed, as above, $(A B C)^{2}$ implies $C^{-2}=A^{2} B^{2}$ so that $C=C^{-4}=\left(A^{2} B^{2}\right)^{2}$. Thus, $G$ is the group $G^{5, p, q}=\left\langle A, B, C \mid A^{p}, B^{q}, C^{5},(A B)^{2},(B C)^{2},(C A)^{2},(A B C)^{2}\right\rangle$ defined by Coxeter [4]. Edjvet and Juhász [9] have recently shown that this group is infinite when $5 \leq p \leq q$, except in the case where $p=q=5$. Since $\pi_{1}(M(2(p+q)-1))$ projects onto $G^{5, p, q}$, it is also infinite.

Remark 9 We can use this argument to show $s=2(p+q)-k$ surgery is infinite provided $k \equiv 1 \bmod 5$. The idea is to add the relator $x^{k-1}$ to form a factor group of $\pi_{1}(M(2(p+q)-k))$. Note that $l$ is of the form $x^{-2(p+q)} \tilde{l}$. After adding $x^{k-1}$, the relator $x^{s} l$ becomes $x^{-1} \tilde{l}$ as before and we arrive at the same group $G^{5, p, q}$ with an additional relator that corresponds to $x^{k-1}$. The relation $x=\tilde{l}$ means that we can equally well think of adding the relator $\widetilde{l}^{k-1}$. However, when we follow this through to the group $G^{5, p, q}$, we see that this amounts to adding the relator $C^{k-1}$. If $5 \mid(k-1)$ this relator is already satisfied in $G^{5, p, q}$. Thus, for such $k, \pi_{1}(M(2(p+q)-k))$ projects onto $G^{5, p, q}$ and is infinite when $5 \leq p \leq q$ and $(p, q) \neq(5,5)$. For example, 
this shows that 18 surgery on the $(-2,5,7)$ knot and 22 surgery on $(-2,5,9)$ are not finite.

Lemma 10 Let $p, q$ be odd with $7 \leq p \leq q$. If $p=7$, let $q \geq 21$ and suppose that $(p, q) \neq(9,9)$. Let $M$ be the complement of the $(-2, p, q)$ pretzel knot. Then $\pi_{1}(M(2(p+q)+1))$ is not finite.

Proof As in the proof of Lemma $8, \pi_{1}(M(2(p+q)+1))$ is $\left\langle a, b, \alpha, \beta \mid \alpha=a^{(p-1) / 2}, \beta=b^{(q-1) / 2}, \beta \alpha b \alpha \beta(a \beta)^{2}(\alpha b)^{2}, \alpha \beta a \beta \alpha(a \beta)^{2}(\alpha b)^{2}\right\rangle$.

Adding the relators $a^{p}, b^{q},\left(\alpha \beta^{-1}\right)^{2}$ yields the group $G^{3, p, q}$. Edjvet and Juhász [9] have shown that this group is infinite under the given conditions on $p$ and $q$.

Remark 11 Edjvet and Juhász determine finiteness of the groups $G^{m, p, q}$ except in the case $(m, n, p) \in\{(3,8,13),(3,7,19)\}$. The argument above suggests that techniques of knot theory may be of use in resolving these outstanding cases. In particular, we've shown that $G^{3,7,19}$ is related to $2(7+19)+1$ surgery of the $(-2,7,19)$ pretzel knot. We will argue below that this surgery is not finite. Can this be used to resolve the open question of the finiteness of $G^{3,7,19}$ ?

\section{The $(-2,5,5)$ pretzel knot}

In this section, $K$ will denote the $(-2,5,5)$ pretzel knot and we will prove

Theorem 12 The $(-2,5,5)$ pretzel knot admits no nontrivial finite surgery.

As a first step, we show that all but one of the boundary slopes of $K$ is detected. By Hatcher and Oertel's [11] algorithm, $K$ has boundary slopes 0, 14, 15, 20 and 22. In the first subsection, using the method of [14], we see that slopes 14 and 15 are detected and we show how that method can be extended to prove that the boundary slopes 20 and 22 are also detected and, moreover, there are at least two ideal points for 20 .

Having shown that all boundary slopes except 0 are detected, we have a good idea of the Culler-Shalen norm of $K$. In the second subsection, we apply this knowledge toward a proof of Theorem 12 . 


\subsection{Ideal points of the complement of the $(-2,5,5)$ pretzel knot}

We now explain how the technique of [14] (with which we assume familiarity) can be used to detect the 14 and 15 slopes. By additional calculation we also show that each of the boundary slopes 20 and 22 are detected with slope 20 having at least two ideal points.

SnapPea [28] gives an ideal triangulation of $S^{3} \backslash K$ with 7 ideal tetrahedra. Let $z_{1}, \ldots, z_{7}$ be the complex parameters of the ideal tetrahedra. We define $z_{k}^{\prime}=1 /\left(1-z_{k}\right)$ and $z_{k}^{\prime \prime}=1-1 / z_{k}$. The gluing equations associated to this ideal triangulation are written in the form

$$
\begin{aligned}
z_{1} z_{2}^{\prime} z_{3} z_{4} z_{5} & =z_{1}\left(1-z_{2}\right)^{-1} z_{3} z_{4} z_{5}=1, \\
z_{1}^{\prime} z_{1}^{\prime \prime} z_{2} z_{4}^{\prime} z_{5}^{\prime} z_{5}^{\prime \prime}\left(z_{6}^{\prime}\right)^{2} z_{6}^{\prime \prime} & =-\left(z_{1}\right)^{-1} z_{2}\left(1-z_{4}\right)^{-1}\left(z_{5}\right)^{-1}\left(z_{6}\right)^{-1}\left(1-z_{6}\right)^{-1}=1, \\
z_{1}^{\prime \prime} z_{2} z_{2}^{\prime \prime} z_{3}^{\prime} z_{3}^{\prime \prime} z_{4}^{\prime}\left(z_{7}^{\prime}\right)^{2} z_{7}^{\prime \prime} & =\left(z_{1}\right)^{-1}\left(1-z_{1}\right)\left(1-z_{2}\right)\left(z_{3}\right)^{-1}\left(1-z_{4}\right)^{-1}\left(z_{7}\right)^{-1}\left(1-z_{7}\right)^{-1}=1, \\
z_{1} z_{2}^{\prime} z_{6} z_{7} & =z_{1}\left(1-z_{2}\right)^{-1} z_{6} z_{7}=1, \\
z_{3} z_{4}^{\prime \prime} z_{5}^{\prime} z_{7} & =-z_{3}\left(z_{4}\right)^{-1}\left(1-z_{4}\right)\left(1-z_{5}\right)^{-1} z_{7}=1, \\
z_{3}^{\prime} z_{4}^{\prime \prime} z_{5} z_{6} & =-\left(1-z_{3}\right)^{-1}\left(z_{4}\right)^{-1}\left(1-z_{4}\right) z_{5} z_{6}=1, \\
z_{1}^{\prime} z_{2}^{\prime \prime} z_{3}^{\prime \prime} z_{4} z_{5}^{\prime \prime} z_{6}^{\prime \prime} z_{7}^{\prime \prime} & =1
\end{aligned}
$$

and the derivatives of the holonomies of the meridian and longitude are given by

$$
\begin{aligned}
M & =z_{1}^{\prime \prime} z_{4}^{\prime}\left(z_{5}\right)^{-1} z_{6}^{\prime}, \\
L & =\left(z_{1}\right)^{-1} z_{1}^{\prime}\left(z_{1}^{\prime \prime}\right)^{-19} z_{2} z_{2}^{\prime}\left(z_{3}^{\prime}\right)^{-1}\left(z_{3}^{\prime \prime}\right)^{-1}\left(z_{4}^{\prime}\right)^{-19} z_{4}^{\prime \prime}\left(z_{5}\right)^{18}\left(z_{5}^{\prime \prime}\right)^{-1}\left(z_{6}^{\prime}\right)^{-19} .
\end{aligned}
$$

Because the product of all the gluing equations is equal to 1 , we can omit the last equation. Each gluing equation can be written in the form

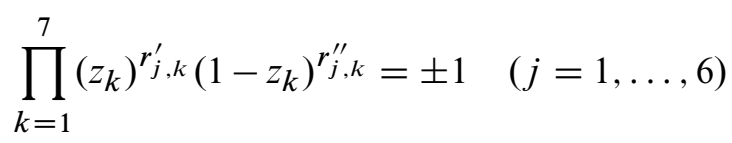

for some integers $r_{j, k}^{\prime}$ and $r_{j, k}^{\prime \prime}$. We denote by $\mathcal{D}$ the affine algebraic set defined by the gluing equations in $(\mathbb{C}-\{0,1\})^{7}$ and call it a deformation variety. For a given point of $\mathcal{D}$, we can construct a $\operatorname{PSL}(2, \mathbb{C})$-representation by using the developing map. It is known that this construction defines an algebraic map from $\mathcal{D}$ to the $\operatorname{PSL}(2, \mathbb{C})$-character variety.

As a sequence of points on $\mathcal{D}$ approaches an ideal point, some $z_{k}$ goes to 0,1 or $\infty$. Let $I=\left\{i_{1}, \ldots, i_{7}\right\}$ where $i_{k}$ is 1,0 or $\infty$. The vector $I$ represents a type of 
degeneration of the ideal tetrahedra. Let

$$
\begin{aligned}
& r(I)_{j, k}=\left\{\begin{array}{lll}
r_{j, k}^{\prime \prime} & \text { if } i_{k}=1, \\
r_{j, k}^{\prime} & \text { if } i_{k}=0, \quad(k=0,1 \ldots, 6) \\
-r_{j, k}^{\prime}-r_{j, k}^{\prime \prime} & \text { if } i_{k}=\infty,
\end{array}\right. \\
& R(I)_{k}=\operatorname{det}\left(\begin{array}{ccccc}
r(I)_{1,1} & \cdots & \widehat{r(I)_{1, k}} & \cdots & r(I)_{1, n} \\
\vdots & & \vdots & & \vdots \\
r(I)_{n-1,1} & \cdots & \frac{r(I)_{n-1, k}}{\cdots} & \cdots(I)_{n-1, n}
\end{array}\right)
\end{aligned}
$$

where the hat means removing the column. We define

$$
d(I)=\left(R(I)_{0},-R(I)_{1}, \ldots, R(I)_{6}\right) .
$$

In [14], it is shown that if all the coefficients of $d(I)$ are positive (or negative), there is a corresponding ideal point of $\mathcal{D}$. The corresponding valuation $v$ satisfies $v\left(z_{k}\right)=d_{k}$. So we can easily compute the values $v(M)$ and $v(L)$. If one of $v(M)$ or $v(L)$ is nonzero, the ideal point of $\mathcal{D}$ gives an ideal point of the $\operatorname{PSL}(2, \mathbb{C})$-character variety and its boundary slope is $-v(L) / v(M)$. Because every PSL(2, $\mathbb{C})$-representation of the fundamental group of a knot complement lifts to a $\operatorname{SL}(2, \mathbb{C})$-representation, we obtain an ideal point of the $\operatorname{SL}(2, \mathbb{C})$-character variety.

By computing $d(I)$ for all $I$, we obtain 6 ideal points of $\mathcal{D}$ satisfying the condition. The computation is shown in Table 1. As in the table, we conclude that the slopes 14 and 15 are detected.

\begin{tabular}{c|c|c|c}
$I$ & $d(I)$ & $(v(M), v(L))$ & boundary slopes \\
\hline$(0,1, \infty, 0,0, \infty, 0)$ & $-(1,2,1,1,1,1,2)$ & $(1,-14)$ & 14 \\
$(\infty, \infty, 0,0, \infty, 0, \infty)$ & $(2,1,1,1,1,2,1)$ & $(-1,14)$ & 14 \\
$(\infty, 1, \infty, 0,0, \infty, 0)$ & $-(1,4,2,4,3,1,6)$ & $(2,-30)$ & 15 \\
$(0,1, \infty, \infty, 0, \infty, 0)$ & $-(4,3,1,1,1,2,1)$ & $(2,-30)$ & 15 \\
$(\infty, 1,0,0, \infty, 0, \infty)$ & $(4,1,3,4,2,6,1)$ & $(-2,30)$ & 15 \\
$(\infty, \infty, 0, \infty, \infty, 0, \infty)$ & $(3,4,1,1,1,1,2)$ & $(-2,30)$ & 15
\end{tabular}

Table 1: Ideal points of $\mathcal{D}$ detected by the method of [14]

2.1.1 Slope 20 (toroidal) If some ideal tetrahedron does not converge to 1,0 or $\infty$, we cannot apply the method of [14] directly. Instead, we make a careful analysis of the nondegenerate ideal tetrahedra. When $\left(z_{1}, z_{2}, z_{3}, z_{4}, z_{5}, z_{6}, z_{7}\right)$ goes to $(*, 0, *, *, *, 1, *)$, there are corresponding ideal points. (The $*$ means that the ideal 
tetrahedron does not become degenerate.) We change the coordinate system of the degenerate ideal tetrahedra by setting

$$
z_{2}=b t, z_{6}=1-f t .
$$

When $t=0$, the points correspond to ideal points. There are two solutions:

$$
\left(z_{1}, z_{3}, z_{4}, z_{5}, z_{7}\right)=\left(-1, \frac{3 \pm \sqrt{-3}}{2}, \frac{3 \pm \sqrt{-3}}{6}, \frac{-1 \pm \sqrt{-3}}{2},-1\right) .
$$

At the ideal point, we have $\left(v\left(z_{2}\right), v\left(1-z_{2}\right)\right)=(1,0),\left(v\left(z_{6}\right), v\left(1-z_{6}\right)\right)=(0,1)$ and $\left(v\left(z_{k}\right), v\left(1-z_{k}\right)\right)=(0,0)$ for $k \neq 2,6$. From the equations (2-1), we have $v(M)=-1$ and $v(L)=20$. So the corresponding boundary slope is $-v(L) / v(M)=$ 20. We remark that the volume near the ideal points approaches $\pm 2.029883 \ldots=$ \pm 2 (the volume of the regular ideal tetrahedron) respectively in the sense of Section 8 of [20]. So the representations near these ideal points are not conjugate. Therefore we conclude that these two ideal points of $\mathcal{D}$ give two different ideal points on the $\operatorname{PSL}(2, \mathbb{C})$-character variety.

We remark that when $\left(z_{1}, z_{2}, z_{3}, z_{4}, z_{5}, z_{6}, z_{7}\right)$ goes to $(1, *, *, *, *, *, 1)$, there are also corresponding ideal points. While they also give a boundary slope of 20 , they appear to be equal to the above two ideal points on the $\operatorname{PSL}(2, \mathbb{C})$-character variety. (In general, the map from $\mathcal{D}$ to the $\operatorname{PSL}(2, \mathbb{C})$-character variety is two to one near an ideal point.)

2.1.2 Slope 22 When $\left(z_{1}, z_{2}, z_{3}, z_{4}, z_{5}, z_{6}, z_{7}\right)$ goes to $(1,1,0, *, 1, *, 0)$, there is a corresponding ideal point. We change the coordinate system for the degenerate ideal tetrahedra by setting

$$
z_{1}=1-a t, z_{2}=1-b t, z_{3}=c t, z_{5}=1-e t^{2}, z_{7}=g t .
$$

The solution is

$$
\left(z_{4}, z_{6}\right)=\left(\frac{1}{2},-1\right)
$$

The corresponding boundary slope is 22 .

When $\left(z_{1}, z_{2}, z_{3}, z_{4}, z_{5}, z_{6}, z_{7}\right)$ goes to $(\infty, 0,1, *, 0,0, *)$, there is also a corresponding ideal point. This is equal to the above ideal point on the character variety.

\subsection{Finite surgeries of $(-2,5,5)$}

Using Hatcher and Oertel's algorithm [11], the boundary slopes of the $(-2,5,5)$ pretzel knot are $0,14,15,20$ and 22. It follows that the Culler-Shalen norm is of the form

$$
\left\|\gamma_{s}\right\|=2\left(a_{1} \Delta(s, 0)+a_{2} \Delta(s, 14)+a_{3} \Delta(s, 15)+a_{4} \Delta(s, 20)+a_{5} \Delta(s, 22)\right)
$$


with $a_{i}$ nonnegative integers. Here $\gamma_{s} \in H_{1}(\partial M ; \mathbb{Z})$ is the class of a curve representing the slope $s \in \mathbb{Q} \cup\left\{\frac{1}{0}\right\}$.

In the previous subsection we showed that all boundary slopes other than 0 are detected and that 20 is detected by two ideal points. It follows that the corresponding constants satisfy $a_{2}, a_{3}, a_{5} \geq 1$ and $a_{4} \geq 2$. However, the following lemma shows that $a_{3} \geq 2$.

Lemma 13 Let $K$ be a hyperbolic knot in $S^{3}$ with Culler-Shalen norm $\|\gamma\|=$ $2 \sum a_{j} \Delta\left(\gamma, \beta_{j}\right)$, the sum being taken over the finite set of boundary slopes, $B=\left\{\beta_{j}\right\}$. If $\beta_{0} \in B$ is represented by a fraction $\frac{u}{v} \in \mathbb{Q} \cup\left\{\frac{1}{0}\right\}$ with $u$ odd then the corresponding constant $a_{0}$ is even.

Proof The A-polynomial of $K$ was defined in [3] as an integral coefficient polynomial $A(l, m)$ in the variables $l$ and $m$. The authors also show that the monomials appearing in $A$ all have $m$ raised to an even power. It follows that the Newton polygon of $A$ has vertices at lattice points $(x, y) \in \mathbb{Z}^{2}$ with $y$ even.

Boyer and Zhang [2] showed that the Newton polygon of $A$ is equivalent to the CullerShalen norm. Specifically, the vectors that connect consecutive vertices of the Newton polygon have the form $a_{j}\left(v_{j}, u_{j}\right)$ where $\frac{u_{j}}{v_{j}}$ is the slope corresponding to the boundary class $\beta_{j}$. Under our hypotheses then, the Newton polygon of $A$ would include vertices of the form $\left(x_{0}, y_{0}\right)$ and $\left(x_{0}+a_{0} v, y_{0}+a_{0} u\right)$. As both $y_{0}$ and $y_{0}+a_{0} u$ are even and $u$ is odd, we must have that $a_{0}$ is even.

Thus, using the results of the previous section, we can assume $a_{2}, a_{5} \geq 1$ and $a_{3}, a_{4} \geq 2$.

Proof of Theorem 12 The algorithm of Hatcher and Oertel [11] shows that the boundary slopes of the $(-2,5,5)$ pretzel knot are $0,14,15,20$ and 22. By Lemma 3, the minimal norm of $K$ is $S=20$. Thus, using Lemmas 2, 4, 5 and 6, a nontrivial finite surgery must lie in the set

$$
\left\{13,16,19,21,23, \frac{31}{2}, \frac{39}{2}, \frac{41}{2}, \frac{43}{2}, \frac{45}{2}\right\} \text {. }
$$

We will use the estimates of the $a_{i}$ 's discussed above to show that none of these has small enough norm to be a finite slope.

If $s \in\left\{\frac{39}{2}, \frac{41}{2}, \frac{43}{2}, \frac{45}{2}\right\}$, then $\left\|\gamma_{s}\right\| \geq 2\left(11 a_{2}+9 a_{3}\right) \geq 58>S+8$. Thus, $s$ is not a finite slope by Lemma 4. Similarly, if $s=\frac{31}{2},\left\|\gamma_{s}\right\| \geq 2\left(3 a_{2}+a_{3}+9 a_{4}+13 a_{5}\right) \geq 72>S+8$. If $s=21$ or 23 , then $\left\|\gamma_{s}\right\| \geq 2\left(7 a_{2}+6 a_{3}\right) \geq 38>S+8$, and these slopes are not finite. Also, $\left\|\gamma_{13}\right\| \geq 2\left(a_{2}+2 a_{3}+7 a_{4}+9 a_{5}\right) \geq 56>S+8$, and $\left\|\gamma_{19}\right\| \geq$ $2\left(5 a_{2}+4 a_{3}+a_{4}+3 a_{5}\right) \geq 36>S+8$, so that 13 and 19 are likewise not finite. 
This leaves only the slope 16 as a candidate for finite surgery. Since $\frac{1}{0}$ is not a boundary slope, it follows from [5, Corollary 1.1.4] and Lemma 3 that $\left\|\frac{1}{0}\right\|=S=20$. We deduce that $\sum_{i=1}^{5} a_{i}=10$. Given our constraints on the $a_{i}$ 's, the smallest possible norm for slope 16 is 44 , which occurs with the choice $\left(a_{1}, a_{2}, a_{3}, a_{4}, a_{5}\right)=(0,1,6,2,1)$. That is, any other distribution of the $a_{i}$ consistent with $\sum_{i=1}^{5} a_{i}=10, a_{2}, a_{5} \geq 1$ and $a_{3}, a_{4} \geq 2$ will result in $\|16\| \geq 44>40=2 S$. Therefore, by Lemma 4,16 is also not a finite slope.

Thus we have shown that the only finite slope of the $(-2,5,5)$ pretzel knot is the trivial slope $\frac{1}{0}$.

\section{The $(-2,5,7)$ and $(-2,5,9)$ pretzel knots}

In this section, we prove two theorems:

Theorem 14 The $(-2,5,7)$ pretzel knot admits no nontrivial finite surgery.

Theorem 15 The $(-2,5,9)$ pretzel knot admits no nontrivial finite surgery.

We prove these theorems using information about detected boundary slopes. For Theorem 15, it suffices to use the slopes detected by the methods of [14]. For Theorem 14, however, we will need to also know something about the number of ideal points for the boundary slope 24 . For this, we will use Ohtsuki's method for calculating the number of ideal points [22; 23]. In the first subsection we give a brief overview of Ohtsuki's approach and find lower bounds for the number of ideal points for the boundary slope 24 of the $(-2,5,7)$ pretzel knot. We then give proofs of our theorems in the subsequent two subsections.

\subsection{Detecting ideal points using Ohtsuki's method}

In this subsection we show that there are at least 8 ideal points for the boundary slope 24 of the $(-2,5,7)$ pretzel knot. These ideal points are detected by using Ohtsuki's method as outlined in [22; 23]. We first briefly recall Ohtsuki's method and then give the calculation of the number of ideal points.

Fix a diagram of a knot $K$ in $S^{3}$ and consider the Wirtinger presentation of $\pi_{1}(M)=$ $\pi_{1}\left(S^{3} \backslash N(K)\right)$ with generators $x_{1}, \ldots, x_{c}$, where $c$ is the number of crossings in the diagram. For $\rho \in \operatorname{Hom}\left(\pi_{1}(M), \operatorname{SL}(2, \mathbb{C})\right)$, we set $X_{i}=\rho\left(x_{i}\right)$ for $i=1, \ldots, c$. Since 
all $X_{i}$ 's are conjugate, their eigenvalues are the same, say $\lambda$ and $\frac{1}{\lambda}$. We assume that $|\lambda|>1$ since we are only interested in the case where $\lambda$ diverges. Let

$$
\left(\begin{array}{c}
x_{i}^{+} \\
1
\end{array}\right) \text { and }\left(\begin{array}{c}
x_{i}^{-} \\
1
\end{array}\right)
$$

denote the eigenvectors of $X_{i}$ corresponding to $\lambda$ and $\frac{1}{\lambda}$ respectively. For each relation $x_{k}=x_{i} x_{j} x_{i}^{-1}$ in the Wirtinger presentation we have the following two equations:

$$
\left(x_{i}^{ \pm}-x_{j}^{-}\right)\left(x_{k}^{ \pm}-x_{j}^{+}\right)-\varepsilon\left(x_{i}^{ \pm}-x_{j}^{+}\right)\left(x_{k}^{ \pm}-x_{j}^{-}\right)=0,
$$

where $\varepsilon=\lambda^{-2}$. Since there are $c$ relations, we have $2 c$ equations of this type. We denote them by $R_{1}, \ldots, R_{2 c}$. Let $\widehat{R}(M)$ denote the algebraic set in $\mathbb{C}^{2 c+1} \cap\{|\varepsilon|<1\}$ with coordinates $\left(x_{1}^{+}, x_{1}^{-}, \ldots, x_{c}^{+}, x_{c}^{-}, \varepsilon\right)$ determined by $R_{1}=\cdots=R_{2 c}=0$. Using a Möbius transformation on $\mathbb{C}$, we can fix three variables in $\left(x_{1}^{+}, x_{1}^{-}, \ldots, x_{c}^{+}, x_{c}^{-}\right)$, which corresponds to taking a slice of $\hat{R}(M)$ with three hyperplanes. We denote this slice by $s(M)$. The character variety $\chi(M)$ is defined to be the set of characters of the $\operatorname{SL}(2, \mathbb{C})$-representations of $\pi_{1}(M)$. There is a canonical map $t: \hat{R}(M) \rightarrow$ $\chi(M) \cap\{|\varepsilon|<1\}$ defined by $t(X)=\operatorname{trace}(X)$. By restricting this map to $s(M)$, we have a surjection $t_{s}: s(M) \rightarrow \chi(M) \cap\{|\varepsilon|<1\}$.

Below, we summarize the algorithm for finding ideal points of $\chi(M)$ in $[22 ; 23]$.

Step 1 Suppose that we wish to detect an essential surface $S$ with a given boundary slope. Choose a set of loops $\ell_{1}, \ldots, \ell_{m}$ in $S^{3} \backslash S$ and make a tree with ends $x_{i}^{ \pm}$ according to [23] (see for instance Section 2 in [23]) in such a way that each of $\ell_{1}, \ldots, \ell_{m}$ has a fixed point when acting on the tree. Note that when we make the tree using Ohtsuki's argument in [23], we use all the equations $R_{1}=\cdots, R_{2 c}=0$ to determine $\widehat{R}(M)$, without omitting three of them. Instead, the three are removed after Step 3 below.

Step 2 Fix three variables using the Möbius transformation. For the other variables, according to the tree obtained in Step 1, we change the coordinates as follows: if the tree suggests that $x_{i}^{\bullet} \equiv x_{j}^{\bullet} \bmod \varepsilon$ then we introduce a new variable $\tilde{x}_{j}^{\bullet}$ in place of $x_{j}^{\bullet}$ such that $x_{j}^{\bullet}=x_{i}^{\bullet}+\tilde{x}_{j}^{\bullet} \varepsilon$, where $\bullet$ represents + or - (and the choice can be made independently for $x_{i}^{\bullet}$ and $x_{j}^{\bullet}$ ). Let $\widetilde{R}_{1}, \ldots, \widetilde{R}_{2 c}$ denote the equations of $R(M)$ in the new variables. These determine an algebraic set in $\mathbb{C}^{2 c+1} \cap\{|\varepsilon|<1\}$ with new coordinates and we denote it by $\widetilde{R}(M)$. The three fixed variables determine a slice of $\widetilde{R}(M)$ and we denote it by $\widetilde{s}(M)$. Note that the above change of coordinates determines a canonical map $\phi: \widetilde{R}(M) \rightarrow \widehat{R}(M)$ and its restriction $\left.\phi\right|_{\widetilde{s}(M)}: \widetilde{s}(M) \rightarrow s(M)$.

Step 3 According to [23, Section 2.4], we make a tunnel near some crossing. Let $R_{1}, R_{2}, R_{3}$ and $R_{4}$ be the equations which have to be removed due to the tunnel. 
Suppose that the generators $x_{i}$ (resp. $x_{j}$ ) of $\pi_{1}(M)$ is separated into $x_{i}$ and $x_{i}^{\prime}$ (resp. $x_{j}$ and $\left.x_{j}^{\prime}\right)$ by the tunnel as in [23, Figure 11].

We will omit the equations $R_{1}, R_{2}$ and $R_{3}$.

Step 4 Let $\hat{R}^{\prime}(M)$ denote the algebraic set in $\mathbb{C}^{2 c+1} \cap\{|\varepsilon|<1\}$ determined by $R_{4}=\cdots=R_{2 c}=0$ and $\widetilde{R}^{\prime}(M)$ denote the one determined by $\widetilde{R}_{4}=\cdots=\widetilde{R}_{2 c}=0$. The change of coordinates in Step 2 determines a canonical map $\phi^{\prime}: \widetilde{R}^{\prime}(M) \rightarrow \widehat{R}^{\prime}(M)$. The three fixed variables determine slices $s^{\prime}(M)$ in $\hat{R}^{\prime}(M)$ and $\widetilde{s}^{\prime}(M)$ in $\widetilde{R}^{\prime}(M)$.

Proposition 16 Let $z$ be an isolated point of the set $\widetilde{s}^{\prime}(M) \cap\{\varepsilon=0\}$ and set $w=\phi^{\prime}(z)$. Suppose that the generators $x_{i}, x_{j} \in \pi_{1}(M)$ separated by the tunnel satisfy $x_{i}^{+} \neq x_{j}^{+}$, $x_{i}^{+} \neq x_{j}^{-}, x_{i}^{-} \neq x_{j}^{+}$and $x_{i}^{-} \neq x_{j}^{-}$in a neighbourhood of $w$. Then $w$ is an ideal point of $s(M)$.

Proof We first show that there is a curve in $\widetilde{s}^{\prime}(M) \backslash\{\varepsilon=0\}$ which converges to $z$ as $\varepsilon \rightarrow 0$. The set $\widetilde{s}^{\prime}(M) \cap\{\varepsilon=0\}$ is an algebraic set in $\mathbb{C}^{2 c+1}$ determined by the equations $R_{4}=\cdots=R_{2 c}=0, \varepsilon=0$ and three equations for making the slice. Hence the number of equations is $2 c+1$. Since the solutions are isolated, we can say that this algebraic set is a locally complete intersection. Hence $\operatorname{dim} \widetilde{s}^{\prime}(M)=1$. This curve cannot be locally contained in $\{\varepsilon=0\}$ because if it were then locally we would have $\operatorname{dim} \widetilde{s}^{\prime}(M)=1$, which contradicts the assumption that $z$ is isolated. Hence this curve satisfies the property claimed.

Now we prove the original assertion. Using the hypotheses and [23, Lemma 2.5], we have $X_{i}=X_{i}^{\prime}$ and $X_{j}=X_{j}^{\prime}$. Thus we can recover the equations $\widetilde{R}_{1}, \widetilde{R}_{2}$ and $\widetilde{R}_{3}$ at each point in a small neighbourhood of $z$. This means that $\widetilde{s}^{\prime}(M)$ locally coincides with $\widetilde{s}(M)$. Hence the curve obtained in the first paragraph can be regarded as a curve in $\widetilde{s}(M) \backslash\{\varepsilon=0\}$ which converges to $z$ as $\varepsilon \rightarrow 0$. By using the map $\left.\phi\right|_{\tilde{s}(M)}: \widetilde{s}(M) \rightarrow s(M)$, we conclude that $w$ is an ideal point of $s(M)$.

Since $\lambda$ diverges at the point $w$ in Proposition 16, using the map

$$
t_{s}: s(M) \rightarrow \chi(M) \cap\{|\varepsilon|<1\},
$$

we conclude that $w$ corresponds to an ideal point of $\chi(M)$.

Remark 17 The generators $x_{i}, x_{j} \in \pi_{1}(M)$ separated by the tunnel both appear in one relation in the Wirtinger presentation. Hence, if the tree in Step 1 is made from local pieces as shown in [23, Figure 4], ie, if there is no degeneration, then we automatically have the condition $x_{i}^{+} \neq x_{j}^{+}, x_{i}^{+} \neq x_{j}^{-}, x_{i}^{-} \neq x_{j}^{+}$and $x_{i}^{-} \neq x_{j}^{-}$. 
Lemma 18 If two solutions $\zeta$ and $\eta$ have different values in the complex ratio

$$
\frac{x_{i}^{\bullet}-x_{k}^{\bullet}}{x_{i}^{\bullet}-x_{\ell}^{\bullet}} / \frac{x_{j}^{\bullet}-x_{k}^{\bullet}}{x_{j}^{\bullet}-x_{\ell}^{\bullet}},
$$

where $x_{i}^{\bullet}, x_{j}^{\bullet}, x_{k}^{\bullet}, x_{\ell}^{\bullet}$ are some ends of generators $x_{1}, \ldots, x_{c}$, then they correspond to different ideal points of $\chi(M)$.

Proof Let $z$ denote the point in $s(M)$ corresponding to the solution $\zeta$ and $w$ denote the one corresponding to $\eta$. Suppose that they correspond to the same ideal point of $\chi(M)$. Then their SL $(2, \mathbb{C})$-representations must be conjugate, ie, there are neighbourhoods $z \in U \subset s(M)$ and $w \in V \subset s(M)$ such that there is a map $\varphi$ of conjugation satisfying $\varphi(z)=w$ and $\varphi(U)=V$. This means that, for each pair of points $u \in U$ and $v=\varphi(u) \in V$, there exists an $\operatorname{SL}(2, \mathbb{C})$-matrix $X$ such that $X \rho_{u}(a) X^{-1}=\rho_{v}(a)$ for every element $a$ in $\pi_{1}(M)$, where $\rho_{u}(a)$ is the $\operatorname{SL}(2, \mathbb{C})$-representation of $a$ at $u \in U$ and $\rho_{v}(a)$ is the one of $a$ at $v=\varphi(u) \in V$. As mentioned in the proof of Lemma C.1 in [23], the Möbius transformation by $X$ sends the ends of generators at $z$ to those at $w$. Thus the complex ratios have to be the same for $\zeta$ and $\eta$.

Lemma 19 There are at least 8 ideal points for the boundary slope 24 of the $(-2,5,7)$ pretzel knot.

Proof Set the generators $a, b, c, d, s_{1}, \ldots, s_{7}, t_{1}, \ldots, t_{9}$ as in left of Figure 1 with identification $a=t_{1}, b=s_{2}, c=t_{8}, d=s_{7}, s_{1}=t_{2}^{-1}$ and $s_{6}=t_{9}^{-1}$. We fix
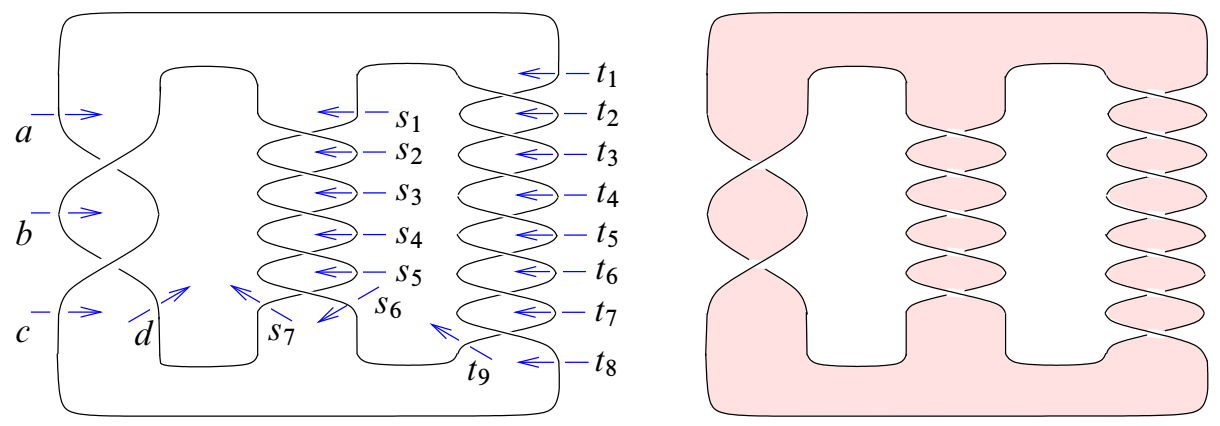

Figure 1: The $(-2,5,7)$ pretzel knot and an essential surface with boundary slope 24

three parameters $t_{1}^{-}=0, t_{1}^{+}=1, t_{3}^{+}=t_{2}^{+}+1$ and change the coordinate $t_{2}^{+}$into $\zeta$ by setting $t_{2}^{+}=1+\zeta$. We first consider Step 1. Since the essential surface with boundary slope 24 is as shown on the right in Figure 1, a tree consisting of 
subtrees of parasol type with the same origin satisfies the necessary condition for corresponding to this surface. For the definition of a subtree of parasol type, see Ohtsuki [22]. We will find 8 ideal points from such a tree. The change of coordinates in Step 2 is done as $b^{-}=a^{+}+r_{1} \varepsilon, c^{-}=b^{+}+r_{2} \varepsilon, d^{-}=c^{+}+r_{3} \varepsilon, s_{1}^{+}=s_{1}^{-}+x_{1}$, $s_{i}^{+}=s_{i-1}^{+}+x_{i}$ for $i=2, \ldots, 7, s_{i}^{-}=s_{i-1}^{+}+y_{i-1} \varepsilon$ for $i=2, \ldots, 7, t_{i}^{+}=t_{i-1}^{+}+p_{i}$ for $i=4, \ldots, 9$ and $t_{i}^{-}=t_{i-1}^{+}+q_{i-1} \varepsilon$ for $i=2, \ldots, 9$, where the $r_{i}$ 's, $x_{i}$ 's $y_{i}$ 's, $p_{i}$ 's and $q_{i}$ 's are nonzero. Using the identification of tangles, we can set $a^{ \pm}=t_{1}^{ \pm}$, $b^{+}=s_{2}^{+}, s_{1}^{-}=\operatorname{solve}\left(b^{-}-s_{2}^{-}, s_{1}^{-}\right), c^{+}=t_{8}^{+}, x_{2}=\operatorname{solve}\left(c^{-}-t_{8}^{-}, x_{2}\right), d^{+}=s_{7}^{+}$, $x_{3}=\operatorname{solve}\left(d^{-}-s_{7}^{-}, x_{3}\right), y_{1}=\operatorname{solve}\left(s_{1}^{+}-t_{2}^{-}, y_{1}\right), x_{1}=\operatorname{solve}\left(s_{1}^{-}-t_{2}^{+}, x_{1}\right)$, $y_{6}=\operatorname{solve}\left(s_{6}^{+}-t_{9}^{-}, y_{6}\right)$ and $x_{6}=\operatorname{solve}\left(s_{6}^{-}-t_{9}^{+}, x_{6}\right)$, where solve is the function which gives the value of the second entry that will make the first entry equal to zero.

The remaining variables are now $\varepsilon, \zeta, r_{1}, r_{2}, r_{3}, x_{4}, x_{5}, x_{7}, y_{2}, \ldots, y_{5}, p_{4}, \ldots, p_{9}$ and $q_{1}, \ldots, q_{8}$, and hence $\widetilde{R}(M)$ is an algebraic set in $\mathbb{C}^{26}$. From the Wirtinger presentation, we have 28 equations $f_{1}^{ \pm}, f_{2}^{ \pm}, g_{i}^{ \pm}$for $i=1, \ldots, 5$ and $h_{i}^{ \pm}$for $i=1, \ldots, 7$ as in Figure 2. Let $F_{1}^{ \pm}, F_{2}^{ \pm}, G_{i}^{ \pm}$for $i=1, \ldots, 5$ and $H_{i}^{ \pm}$for $i=1, \ldots, 7$ denote the leading coefficients of their expansions by $\varepsilon$ after the change of coordinates in Step 2. We omit the equations $G_{1}^{+}, G_{1}^{-}$and $G_{2}^{-}$and solve the others in the following order:

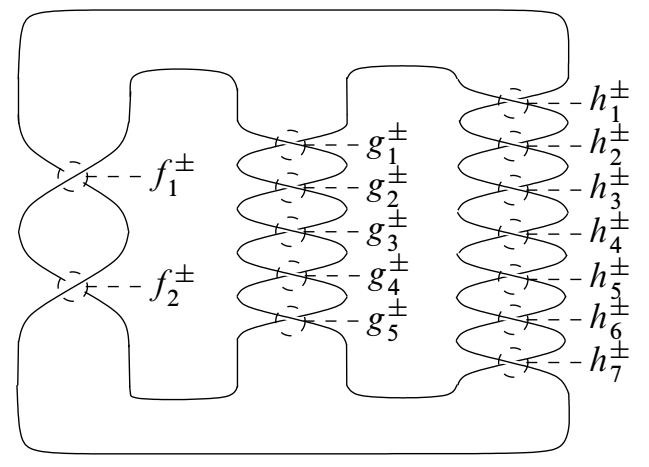

Figure 2: Positions of crossings corresponding to the relations in the Wirtinger presentation

$\left(H_{1}^{+}, q_{1}\right),\left(H_{1}^{-}, q_{2}\right),\left(H_{2}^{-}, q_{3}\right),\left(H_{3}^{-}, q_{4}\right),\left(H_{4}^{-}, q_{5}\right),\left(H_{5}^{-}, q_{6}\right),\left(H_{6}^{-}, q_{7}\right),\left(H_{7}^{-}, q_{8}\right)$, $\left(F_{2}^{+}, r_{2}\right),\left(G_{3}^{+}, y_{3}\right),\left(G_{4}^{+}, y_{4}\right),\left(G_{4}^{-}, y_{5}\right),\left(G_{5}^{+}, x_{4}\right),\left(G_{5}^{-}, r_{3}\right),\left(H_{3}^{+}, p_{5}\right),\left(H_{2}^{+}, p_{4}\right)$, $\left(G_{3}^{-}, x_{7}\right),\left(H_{6}^{+}, p_{8}\right),\left(H_{4}^{+}, p_{6}\right),\left(H_{5}^{+}, p_{7}\right),\left(H_{7}^{+}, p_{9}\right),\left(F_{2}^{-}, x_{5}\right),\left(F_{1}^{+}, r_{1}\right),\left(G_{2}^{+}, y_{2}\right)$, where we again solve by finding the value of the second variable that will make the first zero. Then checking the equation $F_{1}^{-}=0$ we have

$$
\begin{aligned}
0= & \left(\zeta^{8}+8 \zeta^{7}+21 \zeta^{6}+14 \zeta^{5}-19 \zeta^{4}-20 \zeta^{3}+5 \zeta^{2}+2 \zeta+1\right) \\
& \times\left(\zeta^{8}+8 \zeta^{7}+21 \zeta^{6}+14 \zeta^{5}-19 \zeta^{4}-20 \zeta^{3}+7 \zeta^{2}+6 \zeta-1\right) .
\end{aligned}
$$


By using resultant, we can verify that the $r_{i}$ 's, $x_{i}$ 's $y_{i}$ 's, $p_{i}$ 's and $q_{i}$ 's are nonzero for all solutions of this equation. It is also easy to check that the 16 solutions of this equation have 8 different values in the cross ratio

$$
\frac{t_{1}^{+}-t_{2}^{+}}{t_{1}^{+}-t_{1}^{-}} / \frac{t_{3}^{+}-t_{2}^{+}}{t_{3}^{+}-t_{1}^{-}} \text {. }
$$

Hence there are at least 8 ideal points by Lemma 18 .

Remark 20 The methods of the above lemma can also be used to show that there are at least two ideal points for the boundary slope 20 of the $(-2,5,5)$ pretzel knot. In this case, we can find two other ideal points coming from an $r$-curve by the following reason: Let $M$ denote the $(-2,5,5)$ pretzel knot complement and $M_{2,5}$ denote the $(2,5)$ torus knot complement. There is a branched double covering map $M \rightarrow M_{2,5}$ such that the essential surface $S$ with boundary slope 20 is mapped to the essential Möbius band in $M_{2,5}$ with boundary slope 10 . This means that $M$ has an $r$-curve with $r=20$. Since it is shown in [23, Section 5.1] that the boundary slope 10 of $M_{2,5}$ has two ideal points, we can conclude that this $r$-curve also has two ideal points. The existence of these two additional ideal points can also be checked by using Ohtsuki's method for $M$ with a degenerate tree in Step 1. Thus we can conclude that the boundary slope 20 of the $(-2,5,5)$ pretzel knot has at least four ideal points in total.

\subsection{Finite surgeries on $(-2,5,7)$}

In this subsection, we prove Theorem 14.

Proof of Theorem 14 Using [11], the norm of $(-2,5,7)$ is of the form

$$
\begin{aligned}
\|\gamma\|=2\left(a_{1} \Delta(\gamma, 0)+a_{2} \Delta(\gamma, 14)+a_{3} \Delta(\gamma, 15)\right. & +a_{4} \Delta\left(\gamma, \frac{37}{2}\right) \\
& \left.+a_{5} \Delta(\gamma, 24)+a_{6} \Delta(\gamma, 26)\right)
\end{aligned}
$$

where the $a_{i}$ are nonnegative integers. By Lemma 3, the minimal total norm is $S=34$. Using [14], slopes 14,15 and $\frac{37}{2}$ are detected. This implies $a_{2} \geq 1$ and, by Lemma 13, $a_{3}$ and $a_{4}$ must be even, so $a_{3}, a_{4} \geq 2$. As argued in the previous subsection, 24 is detected by 8 ideal points, so $a_{5} \geq 8$.

Using Lemmas 2, 4, 5 and 6 a nontrivial finite surgery must lie in the set

$$
\left\{16,17,18,19,20,23,25,27, \frac{47}{2}, \frac{49}{2}, \frac{51}{2}, \frac{53}{2}\right\} \text {. }
$$


If $s \in\left\{\frac{47}{2}, \frac{49}{2}, \frac{51}{2}, \frac{53}{2}\right\}$, then $\left\|\gamma_{s}\right\| \geq 2\left(19 a_{2}+17 a_{3}+20 a_{4}\right) \geq 186>42=S+8$ and such a slope is not finite. If $s \in\{23,25,27\}$, then $\left\|\gamma_{s}\right\| \geq 2\left(9 a_{2}+8 a_{3}+9 a_{4}\right) \geq 86>$ $42=S+8$ and such a slope is not finite.

For $s \in\{17,19\},\left\|\gamma_{s}\right\| \geq 2\left(5 a_{5}\right) \geq 80>42=S+8$ and these are not finite slopes. If $s \in\{16,18\},\left\|\gamma_{s}\right\| \geq 2\left(6 a_{5}\right) \geq 96>68=2 S$, so these are also not finite. Finally, $\left\|\gamma_{20}\right\| \geq 2\left(6 a_{2}+5 a_{3}+3 a_{4}+4 a_{5}\right) \geq 108>2 S$.

Thus, the only finite slope of the $(-2,5,7)$ knot is the trivial slope $\frac{1}{0}$.

\subsection{Finite surgeries on $(-2,5,9)$}

In this subsection, we prove Theorem 15 .

Proof of Theorem 15 As the argument is quite similar to that used to prove Theorem 12 and Theorem 14, we will omit some details. Using [11] the norm is of the form

$$
\begin{aligned}
\|\gamma\|=2\left(a_{1} \Delta(\gamma, 0)+a_{2} \Delta(\gamma, 14)+a_{3} \Delta(\gamma, 15)\right. & +a_{4} \Delta\left(\gamma, \frac{67}{3}\right) \\
& \left.+a_{5} \Delta(\gamma, 28)+a_{6} \Delta(\gamma, 30)\right),
\end{aligned}
$$

and $S=48$ by Lemma 3. By [14], slopes 14,15 and $\frac{67}{3}$ are detected, so that $a_{2} \geq 1$, and, by Lemma 13, $a_{3}, a_{4} \geq 2$.

Using the lemmas of Section 1, the candidates for a finite slope are

$$
\left\{21,22,23,24,27,29,31, \frac{55}{2}, \frac{57}{2}, \frac{59}{2}, \frac{61}{2}\right\} \text {. }
$$

The half integral surgeries will have norm at least 278 and slopes $27,29,31$ will exceed 130. Also, $\|23\| \geq 58$. These are all more than $S+8$, so none of these slopes are finite.

For 21 we must consider which distribution of the $a_{i}$ 's will give the least value for $\|21\|$. Since $\frac{1}{0}$ is not a boundary slope, we have

$$
24=S / 2=\left\|\frac{1}{0}\right\| / 2=a_{1}+a_{2}+a_{3}+3 a_{4}+a_{5}+a_{6} .
$$

Thus, $\|21\|$ is minimised by the choice $\vec{a}=\left(a_{1}, a_{2}, a_{3}, a_{4}, a_{5}, a_{6}\right)=(0,2,4,6,0,0)$ which results in $\|21\|=124>S+8$, so 21 is not finite. Similarly, we can minimise $\|24\|$ by choosing $\vec{a}=(0,1,2,6,3,0)$. Then $\|24\|=140>2 S$, so 24 is not finite.

Finally, as in Remark $9, \pi_{1}(M(22))$ surjects onto $G^{5,5,9}$ and is therefore not finite. $\square$ 


\section{The 6-theorem}

In this section, let $K$ be a $(-2, p, q)$ pretzel knot with $p, q$ odd and either $7 \leq p \leq q$ or else $p=5$ and $q \geq 11$. We will prove that $K$ admits no nontrivial finite surgeries.

The argument proceeds in two steps: first, we reduce the candidate slopes for finite surgeries to a short list, and then we treat the short list. In the first subsection, we use the 6-theorem of Agol [1] and Lackenby [16] to show that, in case $7 \leq p \leq q$, the candidates for a finite slope of $K$ are the trivial slope $\frac{1}{0}$ and the integral slopes $2(p+q)+k$ with $k=-1,0,1,2$. Then, by Lemma 6 , the boundary slopes [11] $2(p+q)$ and $2(p+q)+2$ are not finite slopes. Lemma 8 shows that $2(p+q)-1$ is not a finite slope while Lemma 10 says the same of the slope $2(p+q)+1$ except for eight cases with $p=7$ or 9 . We will use Culler-Shalen norm arguments to address these cases in Section 4.3.

In the second case, when $p=5$ and $q \geq 11$, the 6-theorem implies that the only candidates for a finite slope of $K$ are $\frac{1}{0}$ and the integral slopes $2(p+q)+k$ with $k=-2,-1,0,1,2,3$. Lemma 6 again eliminates $2 q+10$ and $2 q+12$, while the slopes $2 q+8$ and $2 q+9$ are also ruled out by Lemma 7 and Lemma 8. In Section 4.2 , we will use the Culler-Shalen norm to rule out the slopes $2 q+11$ and $2 q+13$.

\subsection{Applying the 6-theorem}

In this section we shall study exceptional surgeries of the $(-2, p, q)$ pretzel knot complement. Recall that the surgery on a knot $K$ along slope $s$ is called exceptional if $K(s)$ is reducible, toroidal or Seifert fibred or if $\pi_{1}(K(s))$ is finite or not wordhyperbolic. The following theorem was shown by Agol [1] and Lackenby [16].

Theorem 21 Let $M$ be a compact orientable 3-manifold with interior having a complete, finite volume hyperbolic structure. Let $s_{1}, \ldots, s_{n}$ be slopes on $\partial M$, with one $s_{i}$ on each component of $\partial M$. Suppose that there is a horoball neighbourhood $N$ of the cusps of $M \backslash \partial M$ on which each $s_{i}$ has length more than 6 . Then, the manifold obtained by Dehn surgery along $s_{1}, \ldots, s_{n}$ is not exceptional: it is irreducible, atoroidal and not Seifert fibred and has infinite word hyperbolic fundamental group.

By the geometrization theorem, all nonexceptional surgeries yield hyperbolic manifolds. However, for our purposes we only need the conclusion that the filled manifold has infinite fundamental group. We shall apply Theorem 21 to prove the following two propositions. 
Proposition 22 For $p, q \geq 7$ and odd, the $(-2, p, q)$ pretzel knot has at most five exceptional surgeries. If $s$ is an exceptional surgery, it is one of the following: $\frac{1}{0}$, $2(p+q)-1,2(p+q), 2(p+q)+1$ or $2(p+q)+2$.

Proposition 23 For $q \geq 11$ and odd, the $(-2,5, q)$ pretzel knot has at most seven exceptional surgeries. If $s$ is an exceptional surgery, it is one of the following: $\frac{1}{0}$, $2(5+q)+k(k=-2,-1,0,1,2,3)$.

Remark 24 As shown in [18], 2( $p+q)$-surgery is a toroidal surgery and the trivial surgery $\frac{1}{0}$ is also exceptional. However, in general, the other surgeries listed in the propositions may be hyperbolic.

Proof of Proposition 22 Let $L$ be the link consisting of the $(-2,1,1)$ pretzel knot and two trivial link components encircling the $p$-twist and the $q$-twist. (See Figure 3.) By $-1 / k$-surgery and $-1 / l$-surgery along the two trivial link components we get the $(-2,1+2 k, 1+2 l)$ pretzel knot complement in $S^{3}$. We will construct an ideal triangulation of $S^{3} \backslash L$ and find its complete hyperbolic structure. Then we will study cusp shapes of the link complement and apply the 6-theorem to that 3-cusped manifold.

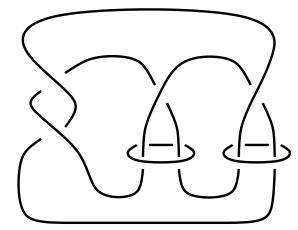

Figure 3: By $-1 / k$-surgery and $-1 / l$-surgery along the trivial link components we get the $(-2,1+2 k, 1+2 l)$ pretzel knot.
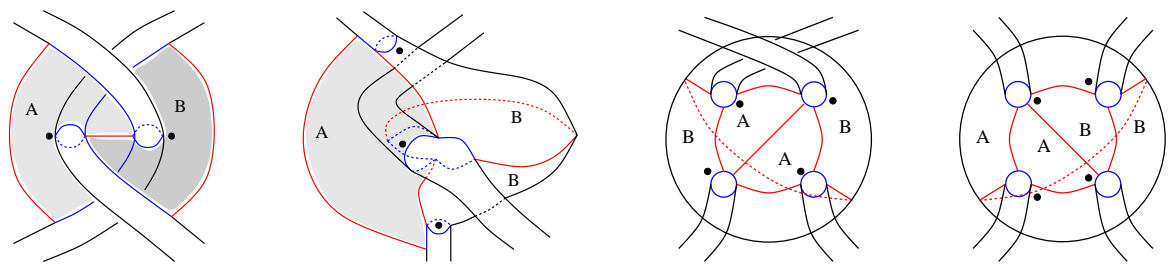

Figure 4: Slice the full twist part.

Take a disk bounding each trivial link component and cut $S^{3} \backslash L$ along these two disks. We also slice the link complement at the full-twist part as shown in Figure 4. The result is Figure 5. In that figure, faces are attached to each other so that the black dots in the 


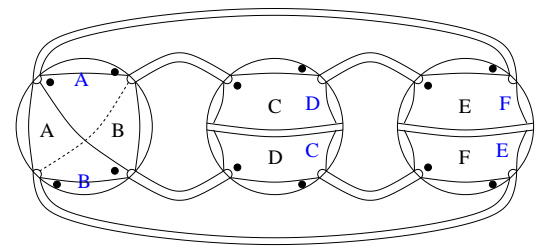

Figure 5: The result of slicing the full twist and along the disks bounded by the trivial components
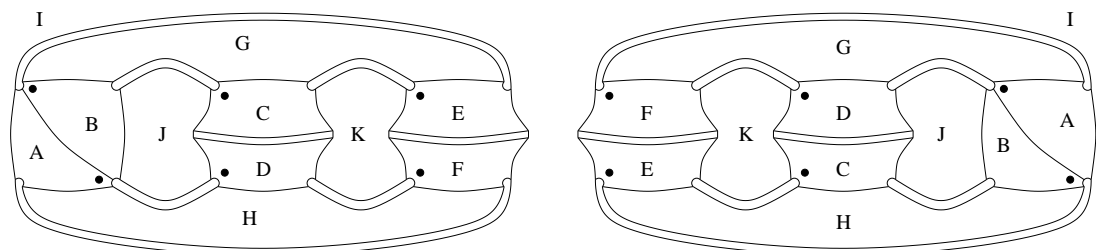

Figure 6: The left is the "upper" side of Figure 5 and the right is the "lower" side of Figure 5. We are viewing these pictures from inside the 3-balls.
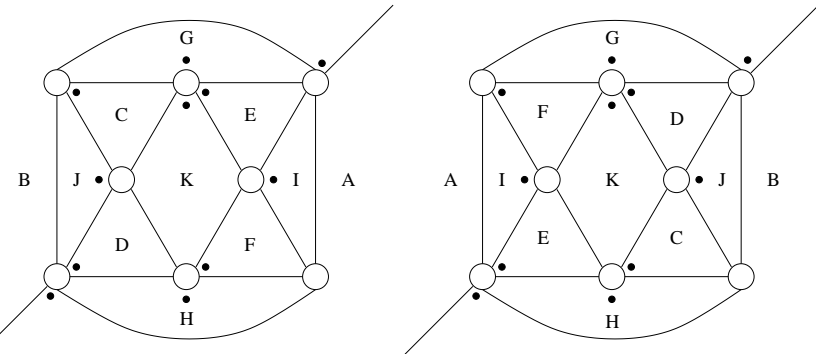

Figure 7: Shrink "fragments of cusps" to small disks.

faces coincide. We then slice the manifold into two balls along the horizontal plane (Figure 6).

Now, shrink fragments of boundary components to small disks. This gives a (topological) ideal polyhedral decomposition of $S^{3} \backslash L$ into two ideal polyhedra (Figure 7). In Figure 7, we change the diagonal edges of the square made of faces $A$ and $I$. We also change the diagonal edge of the square formed by $B$ and $J$ leaving us with two balls with graphs as shown in Figure 8. Glue these two 3-balls along the $K$-faces to arrive at the manifold of Figure 9. We ideally triangulate Figure 9 into ten ideal tetrahedra as shown in Figure 10. 


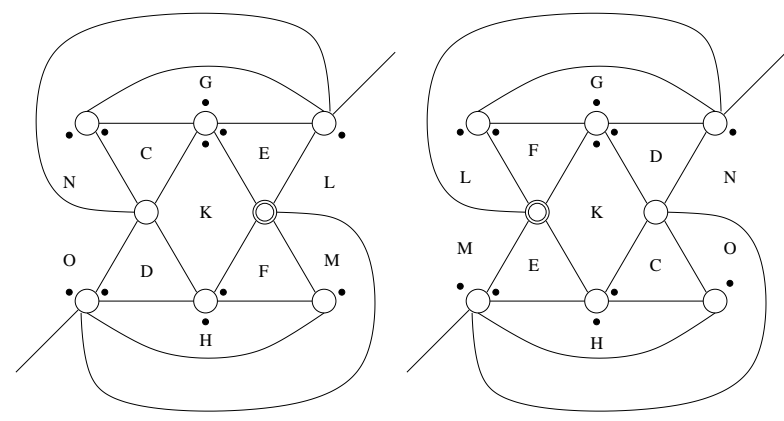

Figure 8: Exchange the diagonals between $A$ and $I$ and also between $B$ and $J$.

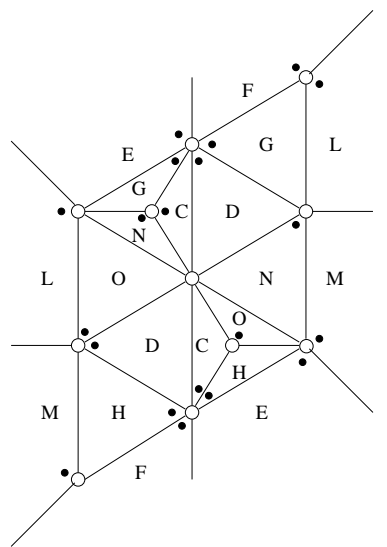

Figure 9: An ideal triangulation of the link complement $S^{3} \backslash L$

For an alternate point of view, we also note this decomposition into ten ideal tetrahedra is a refinement of the ideal polyhedral decomposition that Futer and Guéritaud describe in [10, Section 4].

We can easily observe that each 1-simplex of the ideal triangulation is the edge of 6 ideal tetrahedra. Therefore, if we give a regular ideal tetrahedral structure to each tetrahedron, these ideal tetrahedra will satisfy the gluing equation around each 1simplex. We can see that the torus boundaries have the Euclidean structures shown in Figure 11 and Figure 12. Thus we obtain a complete hyperbolic structure on $S^{3} \backslash L$. If we take a uniform cusp cross-section for each ideal tetrahedron as shown in Figure 13, the cusp cross-section has Euclidean structure as shown in Figure 11 and Figure 12 where the side of each triangle has Euclidean length 1.

We shall apply Theorem 21 to the above cusp cross-section. The universal cover of a cusp is the Euclidean plane, and a basepoint on the torus lifts to a lattice generated 


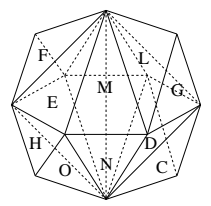

Figure 10: The ideal polyhedron decomposed into 10 ideal tetrahedra

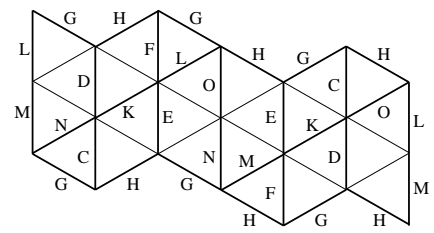

Figure 11: Euclidean structure of torus boundary $((-2,1,1)$ component $)$

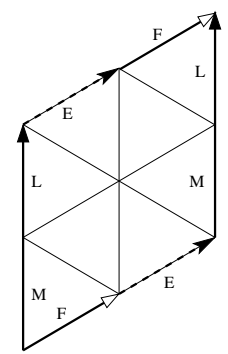

Figure 12: Euclidean structure of torus boundary (trivial component)

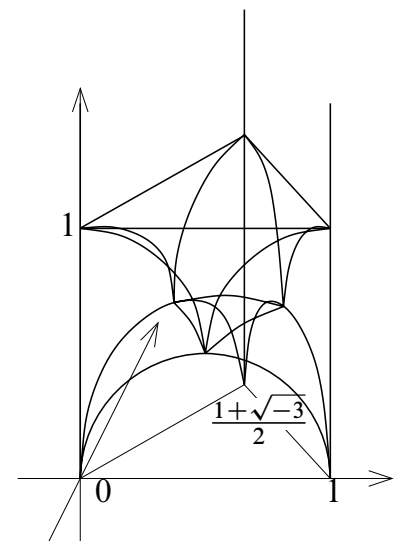

Figure 13: A uniform cusp cross section 
by two complex numbers. Every slope on the torus lifts to a primitive lattice point, where the length of the slope is equal to the Euclidean distance of the lattice point from the origin. For the trivial component of $L$, Figure 12 shows that the lattice is generated by the complex numbers 2 (corresponding to the longitude) and $\sqrt{3} i$ (corresponding to the meridian). Thus the Euclidean length of slope $-1 / k$ is equal to $|\sqrt{-3}-2 k|=\sqrt{3+4 k^{2}}$. For $k \geq 3$, (hence $p, q \geq 7$ ), the slope $-1 / k$ is longer than 6.

At the cusp of the original knot, Figure 13 shows that the lattice giving the Euclidean structure is generated by the complex numbers 2 (corresponding to the meridian) and $-1+3 \sqrt{3} i$ (corresponding to the slope $4 / 1$ ). After $-1 / k$-surgery and $-1 / l$-surgery on the trivial link components, the slope $4 / 1$ becomes $4(k+l)+4=2(p+q)$ because the linking number with each of the trivial link components is equal to 2 . The length of the slope $(2(p+q) m+n) / m$ is equal to $|2 n+(-1+3 \sqrt{3} i) m|=$ $\sqrt{(2 n-m)^{2}+27 m^{2}}$. Therefore, we can realize every surgery on $K$ via a Dehn filling of $L$ along slopes longer than 6 , except $(2(p+q) m+n) / m$ surgery when $(m, n)=(0,1),(1,-1),(1,0),(1,1),(1,2)$. By Theorem 21, this means there are at most 5 exceptional surgeries on $K$.

Proof of Proposition 23 In this case we have to replace the cusp cross-sections in order to enlarge one cusp so that the slope $-1 / 2$ on one of the trivial link components has length greater than 6 . Then the other cusp cross-sections will become smaller. We expand the cusp cross-section corresponding to 5 half twists, by a factor of $\sqrt{2}$. The length of slope $-1 / 2$ on that cusp is now equal to $\sqrt{2\left(3+4 \cdot 2^{2}\right)}=\sqrt{38}>6$. Then the other cusps are contracted by $1 / \sqrt{2}$ (Figure 14).

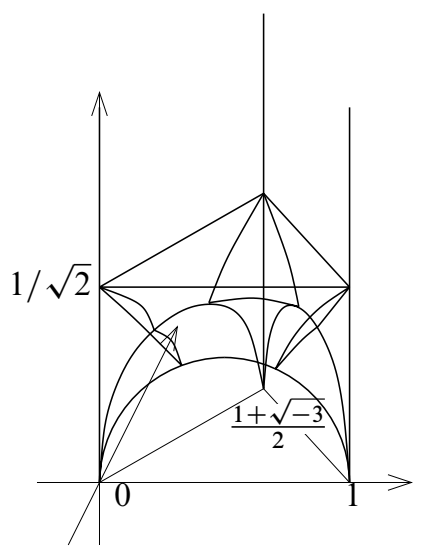

Figure 14: Expanding one cusp cross-section at an ideal vertex 
After this modification of cusp neighbourhoods, the Euclidean lattice for the other trivial link components is generated by the complex numbers $2 / \sqrt{2}=\sqrt{2}$ (corresponding to the longitude) and $\sqrt{3} i / \sqrt{2}=\sqrt{6} i / 2$ (corresponding to the meridian). The Euclidean length of slope $-1 / l$ is equal to $|\sqrt{6} i / 2-\sqrt{2} l|=\sqrt{1.5+2 l^{2}}$. So, for $l \geq 5$, the $-1 / l$ slope is longer than 6 .

At the cusp of the original knot, the Euclidean lattice is generated by $\sqrt{2}$ (corresponding to the meridian) and $-\sqrt{2} / 2+3 \sqrt{6} i / 2$ (corresponding to the slope $4 / 1)$. After $-1 / l$-surgery on the trivial link component, the $4 / 1$-slope becomes $4(2+l)+4=2(5+q)$. The length of the slope $(2(5+q) m+n) / m$ is equal to $|\sqrt{2} n+(-\sqrt{2} / 2+3 \sqrt{6} i / 2) m|=\sqrt{(2 n-m)^{2} / 2+27 m^{2} / 2}$. Thus, except for $(m, n)=(0,1),(1,-2),(1,-1),(1,0),(1,1),(1,2),(1,3)$, every surgery on $K$ can be realized by filling $L$ along slopes longer than 6 . Therefore, there are at most 7 exceptional surgeries.

\subsection{Finite surgeries on the $(-2,5, q)$ pretzel}

In this subsection, we will prove:

Theorem 25 Let $q$ be odd with $q \geq 11$. The $(-2,5, q)$ pretzel knot admits no nontrivial finite surgery.

Proof By Proposition 23, their are seven candidates for finite surgery. Lemma 6 eliminates $2 q+10$ and $2 q+12$ and Lemma 7 and Lemma 8 rule out slopes $2 q+8$ and $2 q+9$.

It remains to examine the slopes $2 q+11$ and $2 q+13$. Since the boundary slopes of the $(-2,5, q)$ pretzel are $0,14,15,\left(q^{2}-q-5\right) /((q-3) / 2), 2 q+10$ and $2 q+12[11]$, the norm is

$$
\begin{aligned}
\|\gamma\|=2\left(a_{1} \Delta(\gamma, 0)\right. & +a_{2} \Delta(\gamma, 14)+a_{3} \Delta(\gamma, 15)+a_{4} \Delta\left(\gamma, \frac{q^{2}-q-5}{(q-3) / 2}\right) \\
& \left.+a_{5} \Delta(\gamma, 2 q+10)+a_{6} \Delta(\gamma, 2 q+12)\right)
\end{aligned}
$$

where the $a_{i}$ are nonnegative integers.

Thus, the norms of $\frac{1}{0}, 2 q+10$ and $2 q+11$ are

$$
\begin{aligned}
\left\|\frac{1}{0}\right\| & =S=2\left(a_{1}+a_{2}+a_{3}+\frac{q-3}{2} a_{4}+a_{5}+a_{6}\right), \\
\|2 q+10\| & =2\left(a_{1}(2 q+10)+a_{2}(2 q-4)+a_{3}(2 q-5)+a_{4}(3 q-10)+2 a_{6}\right), \\
\|2 q+11\| & =2\left(a_{1}(2 q+11)+a_{2}(2 q-3)+a_{3}(2 q-4)+a_{4}(7 q-23) / 2+a_{5}+a_{6}\right) .
\end{aligned}
$$


But then,

$$
\|2 q+11\|-\|2 q+10\|=2\left(a_{1}+a_{2}+a_{3}+\frac{q-3}{2} a_{4}+a_{5}-a_{6}\right) .
$$

In other words, $\|2 q+11\|=\|2 q+10\|+S-4 a_{6}$.

Suppose $a_{i}=0$ for $i \leq 4$. Then $S=2\left(a_{5}+a_{6}\right)$ and $\|2 q+11\|=2\left(a_{5}+a_{6}\right)=$ $S$. As in [7], this would imply that there is a nonintegral boundary slope $r$ with $|2 q+11-r|<1$. As there is no such $r$, we conclude that there exists $i \leq 4$ with $a_{i}>0$. Then $\|2 q+10\| \geq 2\left(2 q-5+2 a_{6}\right)$ and $\|2 q+11\| \geq S+2(2 q-5)>S+8$ so that $2 q+11$ surgery is not finite.

A similar argument shows that $\|2 q+13\|-\|2 q+10\|=3 S-4 a_{6}$. Hence we have $\|2 q+13\| \geq 3 S+2(2 q-5)>S+8$ so that $2 q+13$ is not finite.

\subsection{Finite surgeries on the $(-2, p, q)$ pretzel $(7 \leq p \leq q)$}

In this subsection, we will prove:

Theorem 26 Let $p$ and $q$ be odd with $7 \leq p \leq q$. The $(-2, p, q)$ pretzel knot admits no nontrivial finite surgery.

Proof By Proposition 22, their are five candidates for finite surgery. Lemma 6 eliminates $2(p+q)$ and $2(p+q)+2$ and Lemma 8 rules out the slope $2(p+q)-1$.

So, the only candidate for a nontrivial finite surgery is $2(p+q)+1$ and, by Lemma 10 , we can assume either $p=q=9$ or else $p=7$ and $7 \leq q \leq 19$. However, an argument similar to that used in the previous subsection for $2 q+11$ surgery on the $(-2,5, q)$ pretzel knot shows that these remaining eight cases also do not lead to a finite surgery.

Acknowledgements The second author is supported by MEXT, Grant-in-Aid for Young Scientists (B) (No. 19740029). This research occurred during a visit of the fourth author to Saitama University. He would like to thank the Math Department for their warm hospitality. The fifth author is partially supported by the Ministry of Education, Culture, Sports, Science and Technology, Grant-in-Aid for Scientific Research (C) 18540069. 


\section{References}

[1] I Agol, Bounds on exceptional Dehn filling, Geom. Topol. 4 (2000) 431-449 MR1799796

[2] S Boyer, X Zhang, A proof of the finite filling conjecture, J. Differential Geom. 59 (2001) 87-176 MR1909249

[3] D Cooper, M Culler, H Gillet, D D Long, P B Shalen, Plane curves associated to character varieties of 3-manifolds, Invent. Math. 118 (1994) 47-84 MR1288467

[4] HSM Coxeter, The abstract groups $G^{m, n, p}$, Trans. Amer. Math. Soc. 45 (1939) 73-150 MR1501984

[5] M Culler, C M Gordon, J Luecke, P B Shalen, Dehn surgery on knots, Ann. of Math. (2) 125 (1987) 237-300 MR881270

[6] M Culler, P B Shalen, Varieties of group representations and splittings of 3-manifolds, Ann. of Math. (2) 117 (1983) 109-146 MR683804

[7] N M Dunfield, Cyclic surgery, degrees of maps of character curves, and volume rigidity for hyperbolic manifolds, Invent. Math. 136 (1999) 623-657 MR1695208

[8] M Edjvet, On certain quotients of the triangle groups, J. Algebra 169 (1994) 367-391 MR1297155

[9] M Edjvet, A Juhász, The groups $G^{m, n, p}$, J. Algebra 319 (2008) 248-266 MR2378069

[10] D Futer, F Guéritaud, Angled decompositions of arborescent link complements, Proc. London Math. Soc. 98 (2009) 325-364

[11] A Hatcher, U Oertel, Boundary slopes for Montesinos knots, Topology 28 (1989) 453-480 MR1030987

[12] K Ichihara, I D Jong, Cyclic and finite surgeries on Montesinos knots, Alg. Geom. Topol. 9 (2009) 731-742

[13] M Ishikawa, T W Mattman, K Shimokawa, Exceptional surgery and boundary slopes, Osaka J. Math. 43 (2006) 807-821 MR2303551

[14] Y Kabaya, A method to find ideal points from ideal triangulations, to appear in J. Knot Theory Ramifications arXiv:0706.0971

[15] A Kawauchi, Classification of pretzel knots, Kobe J. Math. 2 (1985) 11-22 MR811798

[16] M Lackenby, Word hyperbolic Dehn surgery, Invent. Math. 140 (2000) 243-282 MR1756996

[17] M Lackenby, R Meyerhoff, The maximal number of exceptional Dehn surgeries arXiv:0808.1176

[18] T W Mattman, The Culler-Shalen seminorms of pretzel knots, PhD thesis, McGill University (2000) Available at http://www.csuchico.edu/ tmattman 
[19] T W Mattman, Cyclic and finite surgeries on pretzel knots, from: "Knots 2000 Korea, Vol. 3 (Yongpyong)”, J. Knot Theory Ramifications 11 (2002) 891-902 MR1936241

[20] W D Neumann, J Yang, Bloch invariants of hyperbolic 3-manifolds, Duke Math. J. 96 (1999) 29-59 MR1663915

[21] U Oertel, Closed incompressible surfaces in complements of star links, Pacific J. Math. 111 (1984) 209-230 MR732067

[22] T Ohtsuki, Ideal points and incompressible surfaces in two-bridge knot complements, J. Math. Soc. Japan 46 (1994) 51-87 MR1248091

[23] T Ohtsuki, How to construct ideal points of $\mathrm{SL}_{2}(\mathbf{C})$ representation spaces of knot groups, Topology Appl. 93 (1999) 131-159 MR1680835

[24] D Rolfsen, Knots and links, Math. Lecture Ser. 7, Publish or Perish, Berkeley, CA (1976) MR0515288

[25] P B Shalen, Representations of 3-manifold groups, from: "Handbook of geometric topology”, (R J Daverman, R B Sher, editors), North-Holland, Amsterdam (2002) 9551044 MR1886685

[26] H F Trotter, Non-invertible knots exist, Topology 2 (1963) 275-280 MR0158395

[27] L Watson, Surgery obstructions from Khovanov homology arXiv:0807.1341

[28] J Weeks, SnapPea Available at http://www.geometrygames.org/SnapPea/

[29] Y-Q Wu, The classification of toroidal Dehn surgeries on Montesinos knots arXiv: math.GT/0610870

Mathematics Department, Temple University

Philadelphia, PA 19122, USA

Mathematical Institute, Tohoku University

Sendai, 980-8578, Japan

Department of Mathematics, Tokyo Institute of Technology

2-12-1 Oh-okayama, Meguro-ku, Tokyo 152-8551, Japan

Department of Mathematics and Statistics, California State University at Chico

Chico, CA 95929-0525, USA

Department of Mathematics, Saitama University

Saitama 338-8570, Japan

dfuter@math.temple.edu, ishikawa@math.tohoku.ac.jp,

kabaya@math.titech.ac.jp, TMattman@Csuchico.edu, kshimoka@rimath.saitama-u.ac.jp

Received: 29 September 2008 